\title{
Individual Modeling Method of a Bone Based on the X-Ray CT Data*
}

\author{
Norio INOU**, Michihiko KOSEKI***, Takeshi SUETSUGU**, \\ Koutarou MAKI**** and Sadayuki UJIHASHI**
}

\begin{abstract}
Reliable stress analysis of a bone requires the precise finite-element model. This study deals with an automated method of an individual finite-element model of a bone based on the X-ray CT data. The method consists of two processes. The first process produces an approximate finite-element model of a bony shape with small tetrahedral elements. For the modeling, various types of tetrahedral elements with almost same volume were provided. The second process transforms the approximate model so that the model is fitted to the outer shape. We applied the method to a human lumber vertebra and a human mandible. The proposed method successfully generated the exact finite-element models of the bones.
\end{abstract}

Key Words : Individual Modeling Method, Finite-Element Model, X-ray CT, Mandible, Vertebra

\section{Introduction}

In the present medical sites, we sometimes have opportunity to make use of three-dimensional measurement systems represented by X-ray CT or MRI. The present application of these systems is limited to medical diagnoses by the picture images. If a method which enables to execute stress analysis of a bone by use of the data from the measurement systems is developed, it will greatly contribute to the medical engineering fields.

The essential problem in the stress analysis is to make an exact mechanical model of a bone as much as possible. That is, it must provide a finite element model that reflects individual bony shape. Moreover, when we must execute stress analyses for many patients, reduction of labor of the individual modeling

* Received 27th November, 1998. Japanese original: Trans. Jpn. Soc. Mech. Eng., Vol. 64, No. 620, p. 12231228 (Received 10th July, 1997)

** Tokyo Institute of Technology, Department of Mechanical and Environmental Informatics, Ookayama, Meguro-ku, Tokyo 152-8552, Japan. E-mail: inou@mei.titech.ac.jp

*** Fujitsu Corporation, Shin-yokohama, Kohoku-ku, Yokohama 222-0033, Japan

**** Showa University, Department of Orthodontics, Kitasenzoku, Ohta-ku, Tokyo 145-0062, Japan will be an important subject.

Automated methods for mechanical elements or industrial tools have already developed and entered the stage of practical application. However, it is difficult to apply the method to a bone because of the intricate shape. For this reason, some research groups have begun to develop automated methods which generate finite element models of bones from the data obtained by X-ray CT or MRI.

R.T Hart et al ${ }^{(1)}$ made a three-dimensional finiteelement model of a human mandible from the $\mathrm{X}$-ray CT data by an automated method and examined the stress distribution produced in the bone. Their automated method is powerful as it directly generates a mandibular model from the X-ray CT data, but there are still some room for improvement because the finite-elements are rather irregular in shape and size as they are monotonously divided by a regular rule. J.H. Keyak and co-researchers ${ }^{(2),(3)}$ proposed another individual modeling methoci. The method is a way to express a bony shape with small cubic elements: As the algorithm of this method is simple and conformable to the computer graphic technique which reconstructs a three-dimensional image from the $\mathrm{CT}$ data, the method will be one of the leading mainstreams in the future. However, the method requires numerous cubic elements for smoothly expressing a 
curved surface of a bone. They presented a finiteelement model of a human femur with about 20000 elements, but the head of the femur was still roughly modeled. If one side of the element decreases to $20 \%$ for smooth modeling, the finite-element model will have more than 2 millions of elements. Such a large model has difficulty in executing stress analysis by the present personal computer.

Müller et al. ${ }^{(4)}$ proposed a new automated modeling method to improve the method by Keyak' s group. Their method is to express a bone with cubic elements, but one cube is divided into five tetrahedral elements. The main improved point is that a curved surface was smoothly expressed by cutting angular parts of the cubes where was close to the surface of the object. They succeeded in generating an exact finite-element model of a cancellous bone. The method is sophisticated, but there is still room to improve because the volumes of elements in a cube are not equal and elements near the surface of the bone tend to be extremely small.

The authors also proposed an individual modeling method that transforms a standard type of model which is provided in advance to the exact model referring to the CT data ${ }^{(5),(6)}$. The method performs efficient stress analysis as it generates an individual model with small number of finite-elements. However, the method has also disadvantage points. One is difficulty in automated modeling because someone must check the finite-element model whether it was properly transformed, and the other one is that we must provide a newly standard type of finite-element model when we apply this method to a different part of bone. Therefore, we propose another individual modeling method focusing on automated modeling this time.

\section{Method of Individual Modeling}

In this chapter, we describe our individual modeling method based on the X-ray CT data. The basic idea of our method is to express a bony shape with various types of tetrahedral finite-elements. We take a different modeling method compared with that of Müller' s group. The largest different point is that our method uses almost equal size of tetrahedral elements to control increase of elements. For the modeling method, we devised a new modeling process. Our method mainly comprises the next two processes.

Process 1. Generation of an approximate model by tetrahedral elements.

Process 2. Transformation of the approximate model.

The first process is to express an object with almost same size of tetrahedral elements. The second process is to transform the approximate model to the exact model. We will explain these processes as follows.

\subsection{Generation of an approximate model}

Process 1 generates an approximate model with almost same size of finite-elements. The modeling data are sliced images of an object with same intervals. To express the shape of the object with flexibility, we provide various types of tetrahedral elements. We classified them into three groups.

Figure 1 shows the first group of elements which are the most basic for the modeling. They are essentially two kinds of elements, but we deal with them as six element types considering dispositions. All elements are in a rectangular box which consists of two rectangular prisms and occupy same volume. Upper and lower planes of the rectangular boxes correspond to planes of CT images. The element type 1, 2, 3 and 4 always include a triangle of the tetrahedron either in the two CT planes. The element type 5 and 6 do not have any triangle in the CT planes, and connect element type 1, 2, 3 and 4 holding a triangle in common.

Aspect ratio of the rectangular box determines the scale of a tetrahedral element. If we set the ratio to be cubic space, the finite-elements become similar regular tetrahedrons which have advantage in high precision computation. The modeling method utilizes a simple rule that a tetrahedral element can produce another tetrahedral element by sharing a common triangle. That is, a new nodal point can produce a new tetrahedral element. Using the simple rule, one element produces a new element one after another within the intended space of the object. The production procedure is executed by the following steps.

Step 1. Place a tetrahedral element at an arbitrary position within the intended space of the object. The placed element is a nucleus of the finite-element
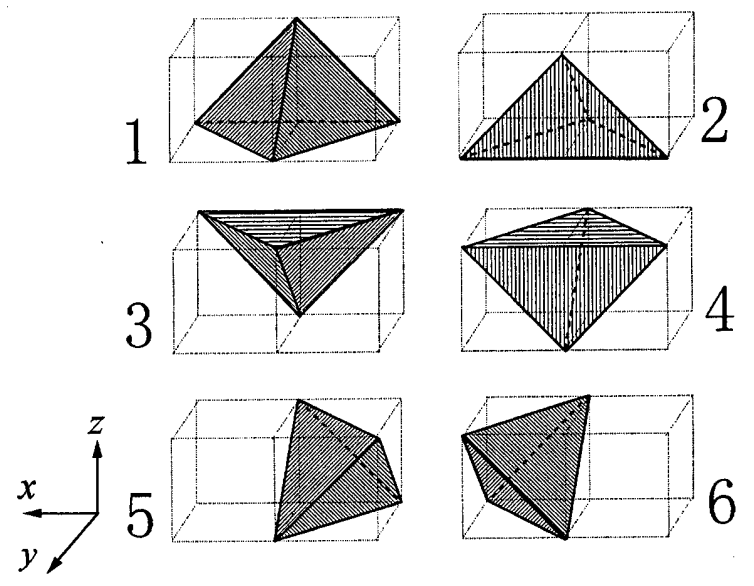

Fig. 1 The furst group of tetrahedral elements for the automated modeling method 


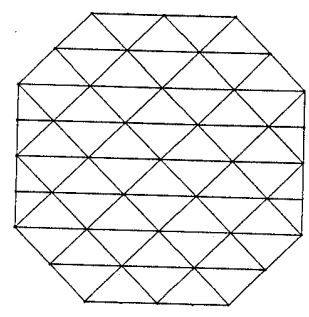

Top view

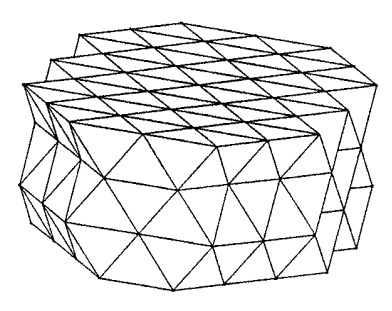

Oblique view
Fig. 2 Generated finite-element model by the process 1. Number of nodal points : 138 , elements : 420

model.

Step 2. Select a tetrahedral element and take notice of one triangle of the element. Then search a proper nodal point from lattice points determined by rectangular prisms regarding the triangle as a base of a new tetrahedral element. There are three possible situations when the point was selected.

Case 1: The position has already been occupied as an apex of other element.

In this case, examine whether there already exists an element in the same space where a new element is being produced. If such an element does not exist, produce a new element there. If it exists, do not produce any element there.

Case 2: The position was not occupied by any tetrahedral element.

In this case, set a new apex and produce a new element.

Case 3 : The position was out of the intended space.

In this case, do not produce a new element.

Step 3. Execute step 2 taking notice of other triangles of the selected element.

Step 4. Execute step 2 and step 3 for all the produced elements and stop the procedure when there is neither apexes nor elements to be executed any more.

Here, we will apply the process 1 to a cylindrical object. Input data for the modeling are several slices of a circular region. Figure 2 shows the result obtained by process 1 . There are many irregularities on the outer surface because apexes of tetrahedral elements can select only lattice points determined by rectangular prisms. The above mentioned process 2 smoothes the irregularities of the approximate model.

\section{2 Transformation of the approximate model}

Process 2 is a kind of molding process so that the approximate model is fitted to the outer shape of the object. This process has an advantage that it does not increase the number of elements by the modification. The molding method is executed by the following steps.

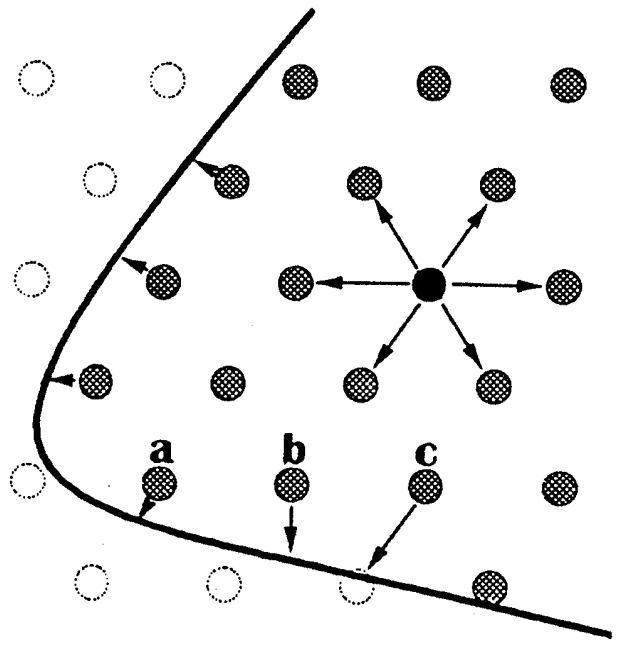

Fig. 3 Transformation of the shape by moving outer side of nodal points

Step 1. Select a nodal point in the approximate model and search the adjacent nodal points in the horizontal plane which is corresponding to a CT image. There are two possible situations by the number of nodal points.

Case 1: The number of adjacent nodes is just six.

In this case, the selected point does not need to move because the point is inside of the object as denoted by a black circle in Fig. 3. Return to step 1 again.

Case 2: The number of nodes is less than six.

In this case, the selected point needs to move as it is an apex of outer surface. Go to step 2.

Step 2. Calculate the moving direction of the nodal point. The direction is determined by summing of the component vectors of adjacent outside nodal points. For example, the arrows in the Fig. 3 show the moving direction of nodal points $\mathrm{a}, \mathrm{b}$ and $\mathrm{c}$ respectively. Then calculate the moving distance of the nodal point to the outline of the object. Return to Step 1 until all nodal points are examined.

Step 3. Transform the approximate model to fit the shape of the object. The transformation is executed by the finite element method under the condition of compulsory displacement using the numerical data obtained by step 2 .

We applied the process 2 to the approximate model. Figure 4 is the result of the transformation by process 2. The finite-element model was modeled without irregularity.

\section{3 Introduction of the second and third groups of elements}

We have successfully shown that the proposed method automatically generates a finite element model of a cylindrical object. However, we still have a problem to be solved when we apply a more 


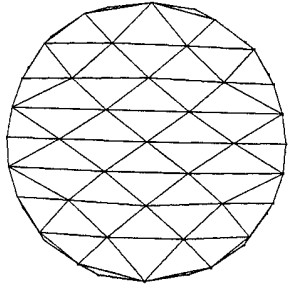

Top view

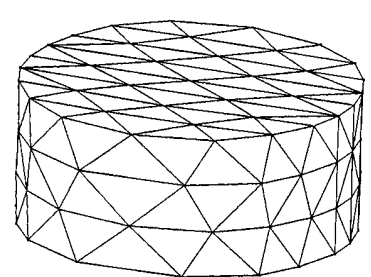

Oblique view
Fig. 4 Generated finite-element model by the process 1 and 2

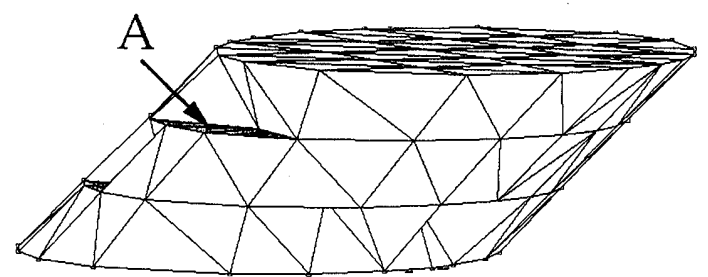

Fig. 5 Modeling result of the oblique cylindrical object by use of the only first group of elements. Number of nodal points : 129 , elements : 398

complicated shape.

Here, we will apply our method to oblique cylindrical shape. Figure 5 shows the result of the modeling by the only first group of elements. There are some defective parts like denoted by A in the model as the first group of elements can not sufficiently fill the inner space of the object. It is important to pack the finite-elements into the intended space without dead space as much as possible. To increase the packing rate, we provided other different types of tetrahedral elements.

Figures 6 and 7 show examples of the additional elements. We call them the second and third groups of elements. The second group has 24 element types which are derived from element type 1,2, 3 and 4 in the first group. Element type 11 to 16 as in Fig. 6 are derived from element type 1 by moving one apex of the element while other three apexes holds a bottom face. Therefore, the volume of the second group of elements is same as that of the first group. The second group of elements are provided for filling spaces where the first group of elements can not fill.

The third group of elements are derived from the second group. Figure 7 shows the example elements which were derived from element type 11. They are connected to the element type 11 having a triangle in common. The third group of elements are more deformed than the second group of elements and does not take same volume as other groups of elements. Usage of the three groups of elements are as follows.

First, we use the first group of elements to fill the space of object. Second, we use the second group of
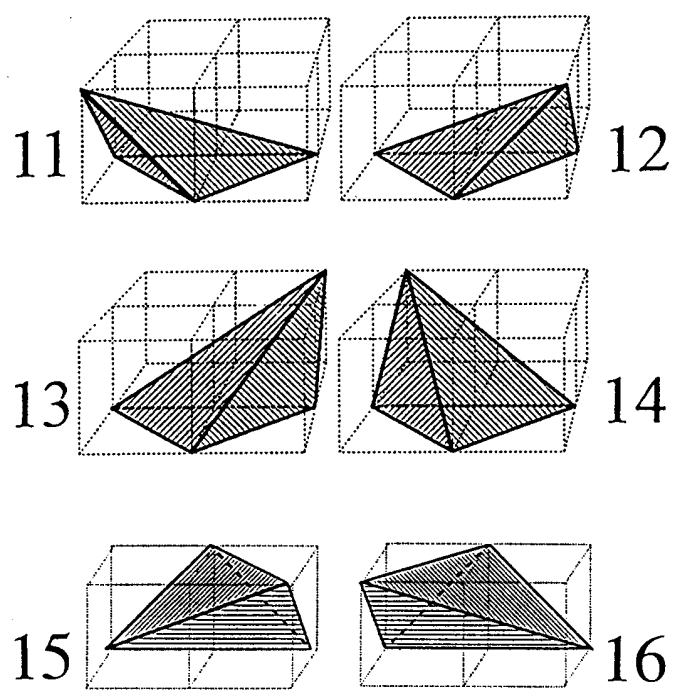

Fig. 6 The second group of elements

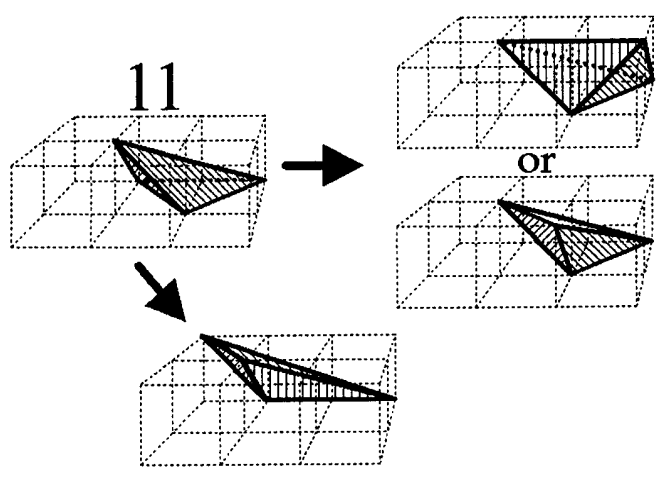

Fig. 7 The third group of elements

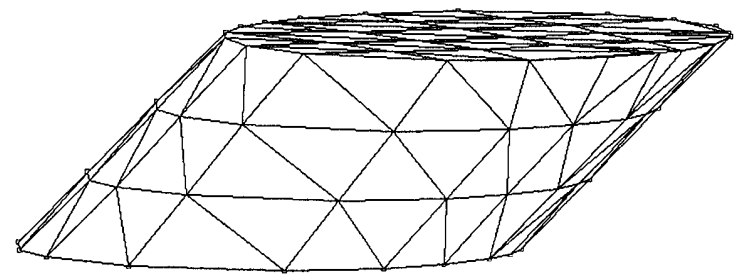

Fig. 8 Modeling result of the oblique cylindrical object by use of the first, sencond and third groups of elements

elements to fill the space where the first group of elements can not fill. Finally, we use the third group of elements to fill the space where the second group of elements can not fill. Figure 8 shows the result of modeling using the three groups of elements applying to the oblique cylindrical model. We successfully obtained the exact finite-element model.

\section{Individual Modeling of a Bone}

For executing process 1 and 2 for the individual modeling, we must extract bony parts from the CT data. First, we will describe the method. 


\section{1 Extraction of bony parts}

For the individual modeling, we used $\mathrm{CT}$ data which were taken at $2 \mathrm{~mm}$ spaces with resolution of $512 \times 512$ pixels. A pixel represents a CT number which reflects degree of nonpermeability coefficient in a material. Figure $9(\mathrm{a})$ shows a original $\mathrm{CT}$ image of a human lumber vertebra. Light part of the image denotes a bone. The $\mathrm{CT}$ number is proportional to the bone density. We can extract bony parts from each CT data utilizing that bony tissue has large CT number compared with soft tissues around the bone. However, it is difficult to extract from the original image directly. Therefore, we introduced the following digital image processing for the extraction.

Figure $9(\mathrm{a}),(\mathrm{b}),(\mathrm{c})$ and $(\mathrm{d})$ show the process of extracting the bony part from each CT image. To emphasize the bony part in the original data as in Fig. 9(a), we transformed the data into several digital values. We set the four kinds of thresholds in this case considering that the outside bone has large CT numbers as it is made with cortical bone and further-
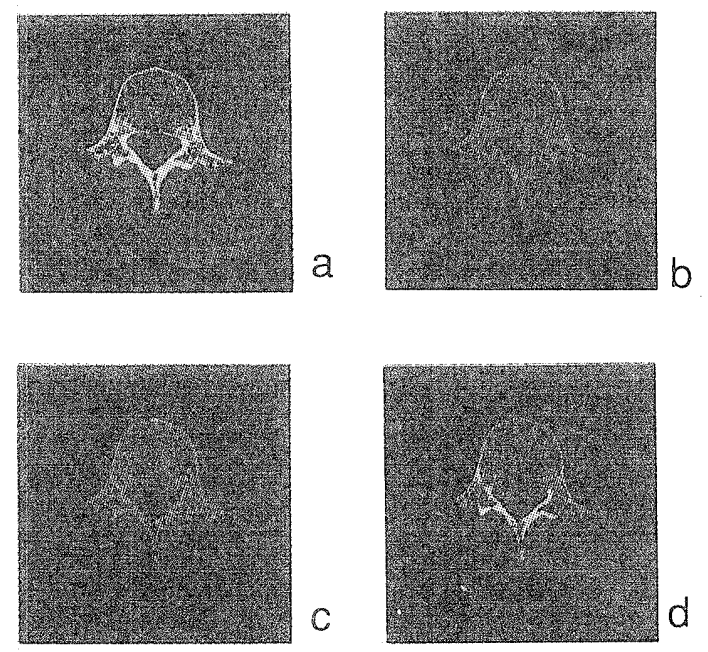

Fig. 9 Extraction of the bony part (a human vertebra)

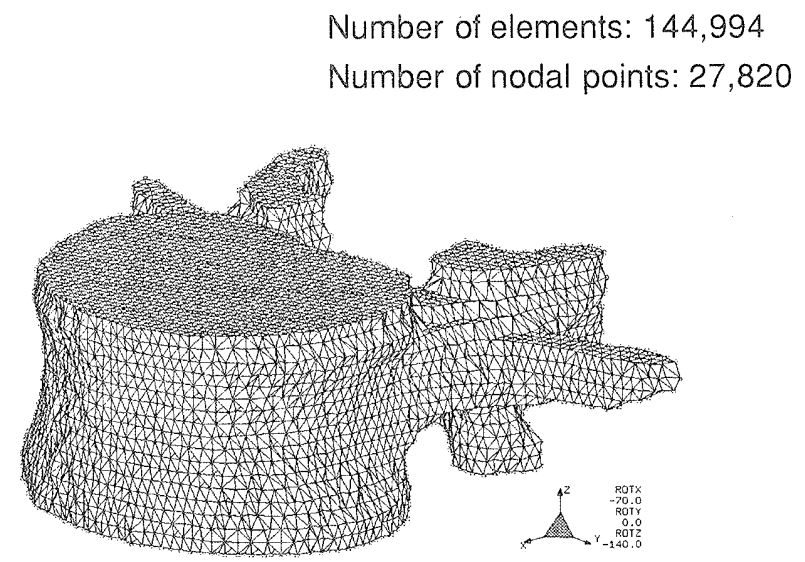

Fig. 10 Generated finite-element model of the human vertebra more, the bone is surrounded with soft tissues which have small CT numbers. Figure $9(\mathrm{~b})$ is the transformed image. From the transformed image, we could easily extract the bony part by removing the circumferential soft tissues. Figure $9(\mathrm{c})$ is the extracted part. Using the image, we can also easily extract the bony outline which is necessary for process 2 . Finally we obtained the bony part by transferring the original data in Fig. $9(\mathrm{a})$ to the extracted part. Figure $9(\mathrm{~d})$ is the final image of the bony part.

\subsection{Modeling of a bone}

Figure 10 is the finite-element model of the human lumber vertebra by the modeling method. Figure 11 is the image of the lumber vertebra reconstructed by an instrument (Voxel Flinger) using the same CT data. We can see that the modeling method successfully generated the exact finite-element model.

In the same way, we made an individual model of the human mandible as in Fig. 12. We can see the successful modeling comparing with the image by the reconstructed three-dimensional image as in Fig. 13.

We used Visual $\mathrm{C}++$ for development of the application software. It took about two hours to generate the model with a personal computer (DEC

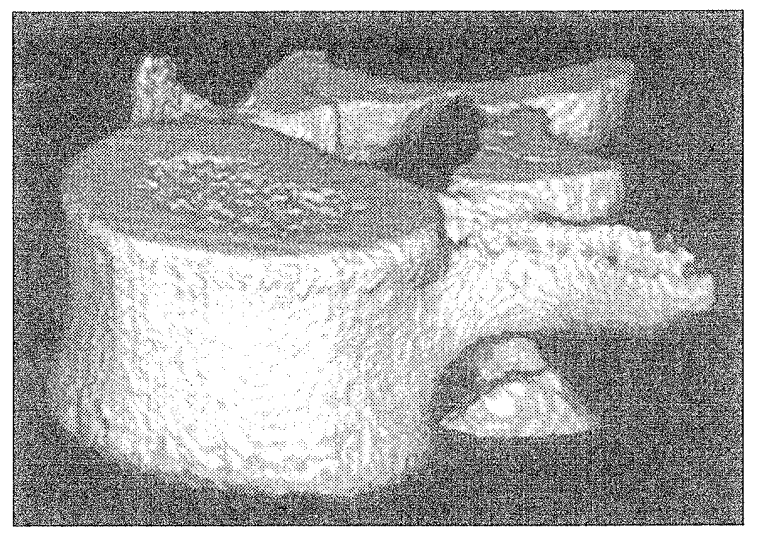

Fig. 11 Three-dimensional image of the same vertebra

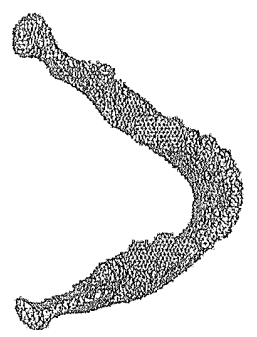

Number of elements: 65,583 Number of nodal points:13,696
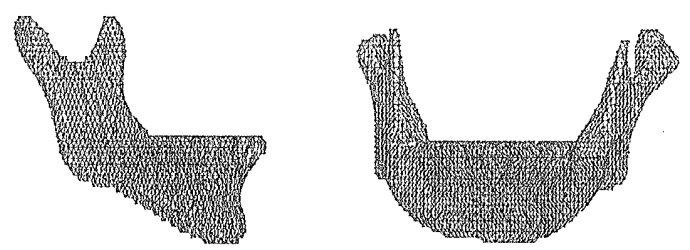

Fig. 12 Generated finite-element model of the mandible 

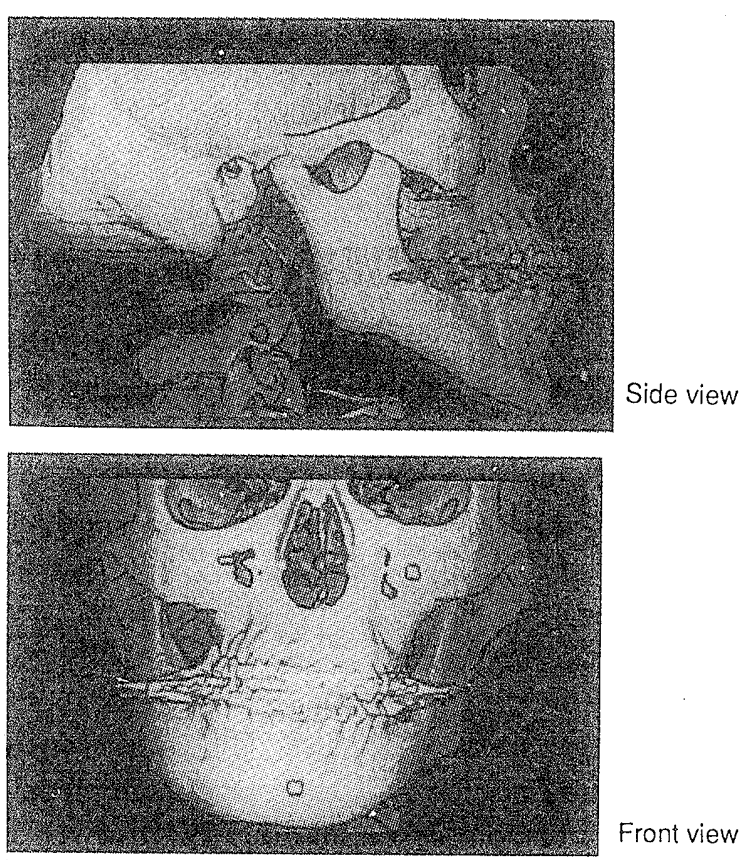

Fig. 13 Three-dimensional image of the same mandible

alpha $433 \mathrm{MHz}$ ) though the time depends the number of finite-elements.

\section{Conclusions}

We proposed an automated modeling method of a bone based on the X-ray CT data and showed that the method generates the exact finite element model with almost same size of tetrahedral elements. The proposed method is useful for not only the human vertebra but also other parts of bones. We have not yet compared our method with that of Müller's group as CT data used for modeling are different. In the next step, we will apply our method to a cancellous bone using the data by micro X-ray CT.

Execution of stress analysis requires material properties of finite-elements and the mechanical conditions. The stress analyses of the human vertebra and mandible will be reported in another paper.

The proposed modeling method is applicable to a three-dimensional display system of a human mandibular movement because the method provides an exact surface model of the individual human mandible. The display system is realized by drawing the images according the movement data obtained by the measurement system of mandibular moment. The details of this study will be reported in other paper.

\section{References}

(1) Hart, R.T., Hennebel, V.V., Thongpreda, N., Buskirk, W. C. and Anderson, R. C., Modelling the Biomechanics of the Mandible (A Three-Dimensional Finite Element Study), J. Biomechanics, Vol. 25 (1992), p. 287-295.

(2) Keyak, J.H., Meager, J.M., Skinner, H.B and Mote, C. D., Automated Three-Dimensional Finite Element Modeling of Bone: a New Method, J. Biomed. Eng., Vol. 12 (1990), p. 389-396.

(3) Keyak, J.H., Fourkas, M.G., Meager, J.M. and Skinner, H.B., Validation of an Automated Method of Three-Dimensional Finite Element Modelling of Bone, J. Biomed. Eng., Vol. 15 (1993), p. 505-509.

(4) Müller, M. and Ruegsegger, P., Three-Dimensional Finite Element Modelling of NonInvasively Assessed Trabecular Bone Structures, Med. Eng. Phys., Vol. 17 (1995), p. 126-133.

(5) Inou, N., Kobayashi, H. and Maki, K., A Modelling Method for an Individual Human Mandible Based on X-ray CT Data, Trans. Jpn. Soc. Mech. Eng., (in Japanese), Vol.60, No. 574 (1994), p. 2078 $-2083$.

(6) Inou, N., Iioka, Y., Fujiwara, H. and Maki, K., Functional Adaptation of Mandibular Bone, Computational Biomechanics, Hayashi, K. and Ishikawa, H. (Eds), (1996) p. 23-42, Springer-Verlag.

(7) Inou, N., Kurami, T., Maki, K. and Ujihashi, S., Three-Dimensional Display System of Mandibular Movement Using X-ray CT Data, World Congress on Medical Physics and Biomedical Engineering (Nice/France), Abstracts book part 1, (1997) p. 613. 